\title{
Kikuchi-Fujimoto disease: A rare cause of lymphadenopathy and fever
}

\author{
Ana Vilaça, Vanessa Palha, Teresa Pimentel
}

\begin{abstract}
Introduction: Kikuchi-Fujimoto disease (KFD) was originally described in a young woman and is a rare, benign and self-limited condition of unknown cause, usually characterized by cervical lymphadenopathy and fever. The diagnosis of KFD is made by lymph node biopsy and treatment is only symptomatic, with signs and symptoms usually resolving within one to six months. Case Report: A 31-year-old female, rabbit breeder, presented with complaints of fever, facial and hand skin lesions for 10 days. Physical examination revealed nose, malar, scalp, fingers and pinna erythematous papules; also painful bilateral cervical and axillary lymphadenopathy. Laboratory studies showed normocytic/normochromic anemia (hemoglobin $10.4 \mathrm{~g} / \mathrm{dL}$ ), leukopenia (white blood cell count $2.2 \times 10^{3} \mathrm{u} / \mathrm{L}$ ), neutropenia 44.4\% and elevated erythrocyte sedimentation rate $31 \mathrm{~mm} / \mathrm{h}$. Serology for infectious agents, microbiology and autoimmune blood work studies were negative. Computed tomography scan of the neck revealed small cervical lymphadenopathy and enlarged axillary lymphadenopathy. She underwent biopsy of a right axillary lymph node, which revealed
\end{abstract}

Ana Vilaça ${ }^{1}$, Vanessa Palha ${ }^{1}$, Teresa Pimentel ${ }^{2}$

Affiliations: ${ }^{1} \mathrm{MD}$, Resident, Internal Medicine Department, Hospital Braga, Braga, Portugal; ${ }^{2} \mathrm{MD}$, Consultant, Internal Medicine Department, Hospital Braga, Braga, Portugal.

Corresponding Author: Ana Vilaça, Travessa S. Pedro, $n^{\circ} 4$, Este S. Pedro, Braga, Portugal, 4715-434; Emil: ana.vilaca. rodrigues@gmail.com

Received: 08 May 2017

Accepted: 22 July 2017

Published: 10 July 2017
Kikuchi lymphadenitis xanthomatous type. Skin biopsy showed unspecified perivascular lymphocytic infiltrate. On a three-month follow-up visit, she continued to be afebrile, had improved white cell blood count and had only small right axillary lymphadenopathy visible on ultrasound. Skin lesions had disappeared. Conclusion: Kikuchi-Fujimoto disease should be considered in the differential diagnosis when a young woman presents with fever and cervical lymphadenopathy. Despite its benign and selflimited nature, patients should be followed for a few years since recurrence is common and some patients may develop autoimmune disorders.

Keywords: Fever, Histiocytic necrotizing lymphadenitis, Kikuchi-Fujimoto disease, Lymphadenopathy

\section{How to cite this article}

Vilaça A, Palha V, Pimentel T. Kikuchi-Fujimoto disease: A rare cause of lymphadenopathy and fever. Case Rep Int 2017;6:31-34.

Article ID: 100039CRINTAV2017

$$
* * * * * * * * *
$$

doi:10.5348/crint-2017-38-CR-7

\section{INTRODUCTION}

Kikuchi-Fujimoto disease (KFD) was described in 1972 in Japan by Kikuchi and Fujimoto [1-3]. It has been reported in several countries since then. Also known as histiocytic necrotizing lymphadenitis, is a rare, benign and 
self-limited condition of unknown cause. It was originally described in a young woman predominantly in their second or third decades of life. Kikuchi-Fujimoto disease is usually characterized by cervical lymphadenopathy and fever. More rarely, patients with KFD can also present leukopenia, hepatosplenomegaly and complaints of fatigue, rash, joint pain and weight loss [4].

The diagnosis of KFD is made by lymph node biopsy, based on histopathological findings. Although, is important to consider differential diagnosis in order to exclude more serious conditions such as lymphoma, infections and autoimmune disorders. Normally, the disease has a good prognosis without therapy. Nonetheless, if necessary, we can use analgesics and nonsteroid anti-inflammatory drugs, in order to symptomatic treatment $[4,5]$.

\section{CASE REPORT}

A 31-year-old woman, rabbit breeder, with history of recent contact with dead rats, presented to the emergency department with complaints of fever (axillary temperature $38^{\circ} \mathrm{C}$ ), facial and hand skin lesions for 10 days. She also presented healing oral ulcers. Physical examination showed nose (Figure 1A), malar, scalp, fingers (Figure 1B) and pinna (Figure $1 \mathrm{C}-\mathrm{D}$ ) erythematous papules. It is also palpable bilateral cervical and axillary lymphadenopathy. These lymphadenopathies were painful. Laboratory examinations revealed normocytic/normochromic anemia (hemoglobin $10.4 \mathrm{~g} / \mathrm{dL}$ ), leukopenia (white blood cell count $2.2 \times 10^{3} \mathrm{u} / \mathrm{L}$ ), neutropenia $44.4 \%$ and elevated erythrocyte sedimentation rate $31 \mathrm{~mm} / \mathrm{h}$. The patient was admitted to the inpatient floor for further study. As the patient had contact with dead rats and is a rabbit breeder, it was performed empiric treatment with doxycycline. Serology for infectious agents, microbiology and autoimmune blood work studies were negative. It was performed CT scans of the neck, chest, abdomen and pelvis, which revealed small cervical lymphadenopathy and enlarged axillary lymphadenopathy. During admission time no fever was registered and leucopenia was maintained with inversion of the white cell blood count. She underwent ultrasound guided histological fine needle biopsy of a right axillary lymph node. The histopathological examination revealed Kikuchi lymphadenitis xanthomatous type (Figure 2A). Skin biopsy of the lesions showed unspecified perivascular lymphocytic infiltrate (Figure 2B). At this point, doxycycline was suspended and it was prescribed analgesics to symptomatic treatment. After one week, the patient remains afebrile and was discharged home. On a three-month follow-up visit, she continued to be afebrile, had improved white cell blood count and had only small right axillary lymphadenopathy visible on ultrasound. Skin lesions was disappeared.
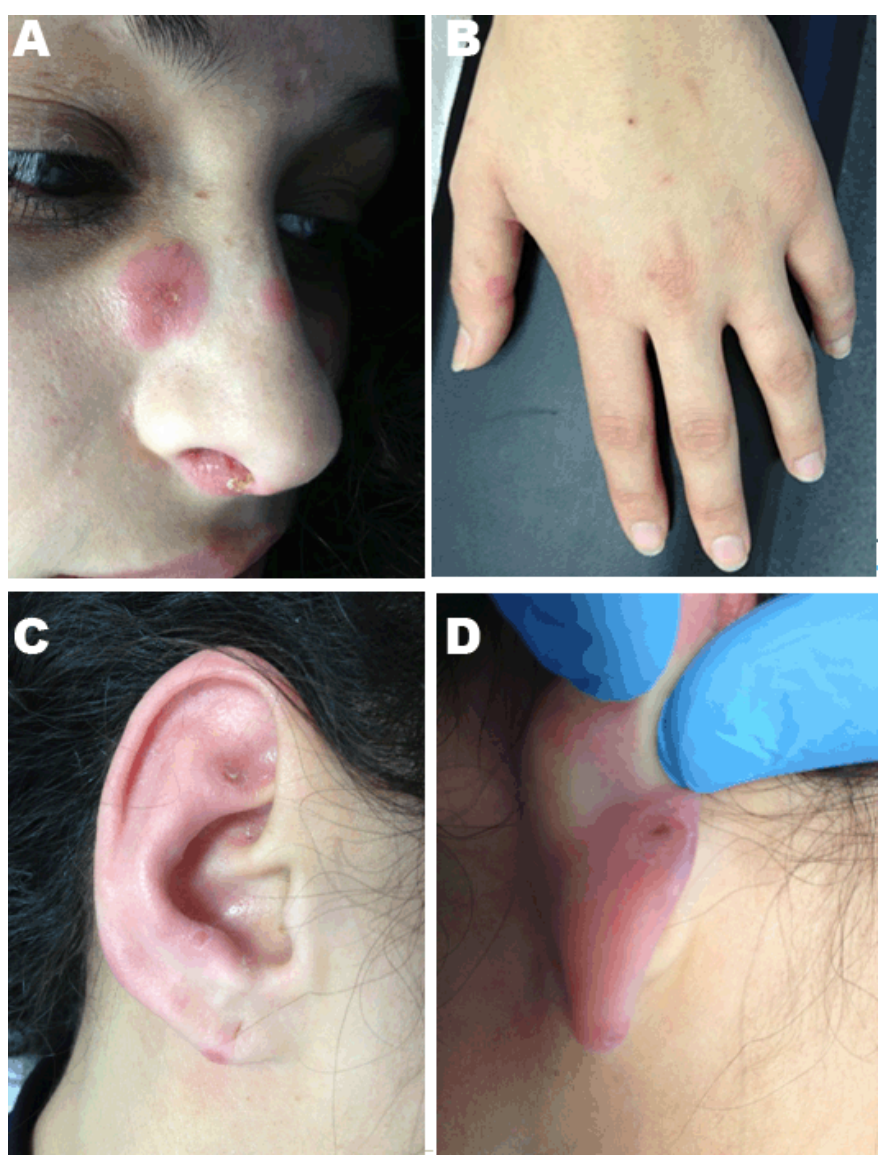

Figure 1: (A) Nose erythematous papules, (B) Fingers erythematous papules, (C) Pinna erythematous macules, (D) Pinna erythematous papules.

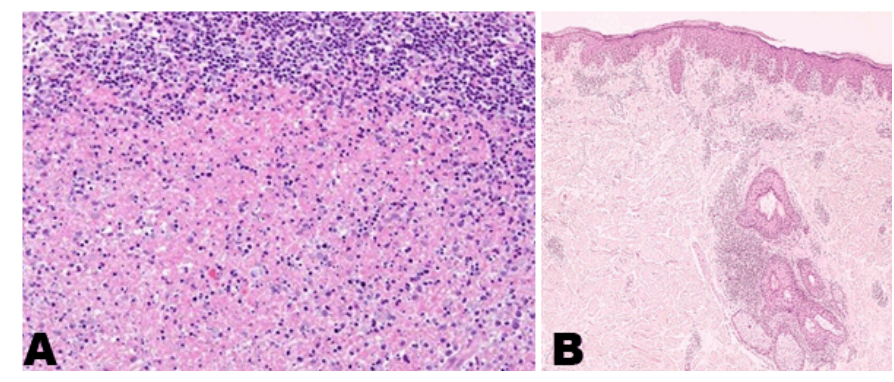

Figure 2: (A) Lymph node biopsy: Areas of apoptotic necrosis, with abundant proliferation of histiocytes and plasmacytoid monocytes in the absence of neutrophils (H\&E stain, x200), and (B) Skin biopsy: Superficial and deep perivascular lymphocytic infiltrate with slight vacuolization of the basal layer of the epidermis (H\&E stain, x400).

\section{DISCUSSION}

Kikuchi-Fujimoto disease should be considered in the differential diagnosis when a young woman presents with fever and cervical lymphadenopathy. In less than $40 \%$ of cases, KFD can also be characterized by rash. Our patient is, in fact, included in these $40 \%$, since at the initial presentation she had erythematous papules at the nose, pinna and fingers (Figures $1 \mathrm{~A}-\mathrm{D}$ ). From 
the dermatological point of view it have been described lesions in the form of macules, papules, plaques, nodules or ulcers on the upper body (face, arms and trunk) [6, 7]. Skin biopsy of the lesions usually shows perivascular infiltrates superficial and deep, edema in the dermis papillary or vacuolization in the layer epidermal basal cells [8].

The nature of this disease remains unclear. Certain causative organisms have been proposed. These include Epstein-Barr virus, human $\mathrm{T}$ cell leukemia virus type 1 , human herpesvirus type 6, B19 parvovirus, cytomegalovirus, Brucella, Yersinia enterocolitica, and parainfluenza vírus [9]. It is suggested that patients receive a systemic survey and follow-up to evaluate subsequent development of SLE, because of the association with systemic lupus erythematosus (SLE) [10, 11]. This autoimmune mechanism remains unclear. Nevertheless, during the past years, some theories were developed. One theory involves molecular mimicry, in which infectious agents that closely resemble a host peptide affect the ability of $\mathrm{T}$ cells to detect self from nonself $[12,13]$. Some authors also believe that apoptotic cells can be the source of the autoantigens of SLE [14].

Since KFD have many symptoms similar to malignant lymphoma, such as Burkitt's lymphoma and Hodgkin's disease, can be often misdiagnosed in the first approach of the patient. Differential diagnosis should always be performed, including infectious lymphadenitis (EBV, Herpes, Bartonella henselae, or Toxoplasma). The correct diagnosis of this disorder requires always the histopathological study of the biopsy $[15,16]$.

Recurrent and fatal cases of KFD are extremely rare. In spite of that, a few had been reported $[11,17,18]$.

A specific treatment for KFD is currently unavailable because of its unknown etiology. Most of the times the KFD is self-limited, resolving in less than six months with symptomatic treatment. Corticosteroid therapy is used only for the most severe cases [4].

\section{CONCLUSION}

In conclusion, despite its benign and self-limited nature, the authors highlight the importance of a precise diagnosis and also the importance of follow-up of these patients for a few years. Recalling our case report, the authors also emphasize the fact that patients with Kikuchi-Fujimoto disease may present non-typical cutaneous lesions, which are important in differential diagnoses.

$* * * * * * * * *$

\section{Author Contributions}

Ana Vilaça - Substantial contributions to conception and design, Acquisition of data, Analysis and interpretation of data, Drafting the article, Revising it critically for important intellectual content, Final approval of the version to be published

Vanessa Palha - Acquisition of data, Analysis and interpretation of data, Revising it critically for important intellectual content, Final approval of the version to be published

Teresa Pimentel - Substantial contributions to conception and design, Analysis and interpretation of data, Revising it critically for important intellectual content, Final approval of the version to be published

\section{Guarantor}

The corresponding author is the guarantor of submission.

\section{Conflict of Interest}

Authors declare no conflict of interest.

\section{Copyright}

(C) 2017 Ana Vilaça et al. This article is distributed under the terms of Creative Commons Attribution License which permits unrestricted use, distribution and reproduction in any medium provided the original author(s) and original publisher are properly credited. Please see the copyright policy on the journal website for more information.

\section{REFERENCES}

1. Moreira A, Louro F, Martins M, Duarte AP. Kikuchi and Fujimoto disease. [Article in Portuguese]. Acta Med Port 2000 Jan-Apr;13(1-2):43-7.

2. Snow RL, Ferry JA. Case records of the massachusetts general hospital. Weekly clinicopathological exercises. Case 5-1997. A 24-year-old woman with cervical lymphadenopathy, fever, and leukopenia. N Engl J Med 1997 Feb 13;336(7):492-9.

3. Reichert A, Correia T, Freitas O, Almeida T, Rosado L. Kikuchi-Fujimoto disease. [Article in Portuguese]. Acta Med Port 2005 May-Jun;18(3):231-4.

4. Hutchinson CB, Wang E. Kikuchi-Fujimoto disease. Arch Pathol Lab Med 2010 Feb;134(2):289-93.

5. Meyer O. Kikuchi disease. [Article in French]. Ann Med Interne (Paris) 1999 Apr;150(3):199-204.

6. Yasukawa K, Matsumura T, Sato-Matsumura KC, et al. Kikuchi's disease and the skin: Case report and review of the literature. Br J Dermatol 2001 Apr;144(4):885-9.

7. Kim KJ, Jee MS, Chang SE, et al. Kikuchi-Fujimoto disease with papulopustular skin manifestations. Clin Exp Dermatol 2003 Mar;28(2):142-4.

8. Spies J, Foucar K, Thompson CT, LeBoit PE. The histopathology of cutaneous lesions of Kikuchi's disease (necrotizing lymphadenitis): A report of five cases. Am J Surg Pathol 1999 Sep;23(9):1040-7.

9. Menasce LP, Banerjee SS, Edmondson D, Harris M. Histiocytic necrotizing lymphadenitis (KikuchiFujimoto disease): Continuing diagnostic difficulties. Histopathology 1998 Sep;33(3):248-54.

10. Hsiang-Cheng C, Jenn-Huang L, Guo-Shu H, et al. Systemic lupus erythematosus with simultaneous onset of Kikuchi-Fujimoto's disease complicated with antiphospholipid antibody syndrome: A case report and review of the literature. Rheumatol Int 2005 
May;25(4):303-6.

11. Leyral C, Camou F, Perlemoine C, Caubet O, Pellegrin JL, Viallard JF. Pathogenic links between Kikuchi's disease and lupus: A report of three new cases. [Article in French]. Rev Med Interne 2005 Aug;26(8):651-5.

12. Marrack P, Kappler J, Kotzin BL. Autoimmune disease: Why and where it occurs. Nat Med 2001 Aug;7(8):899-905.

13. Gross DM, Forsthuber T, Tary-Lehmann M, et al. Identification of LFA-1 as a candidate autoantigen in treatment-resistant Lyme arthritis. Science 1998 Jul 31;281(5377):703-6.

14. Botto M. Links between complement deficiency and apoptosis. Arthritis Res 2001;3(4):207-10.
15. Bosch X, Guilabert A. Kikuchi-Fujimoto disease. Orphanet J Rare Dis 2006 May 23;1:18.

16. Kucukardali Y, Solmazgul E, Kunter E, Oncul O, Yildirim S, Kaplan M. Kikuchi-Fujimoto disease: Analysis of 244 cases. Clin Rheumatol 2007 Jan;26(1):50-4.

17. Bosch X, Guilabert A, Miquel R, Campo E. Enigmatic Kikuchi-Fujimoto disease: A comprehensive review. Am J Clin Pathol 2004 Jul;122(1):141-52.

18. Chan JK, Wong KC, Ng CS. A fatal case of multicentric Kikuchi's histiocytic necrotizing lymphadenitis. Cancer 1989 May 1;63(9):1856-62.
Access full text article on other devices

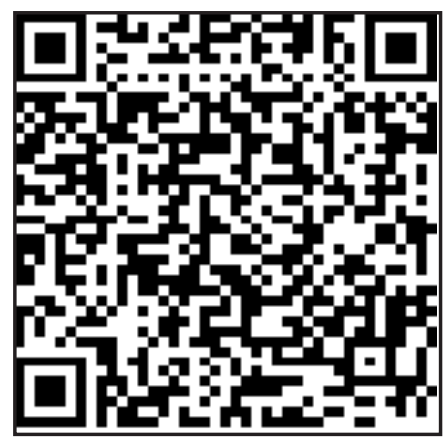

Access PDF of article on other devices

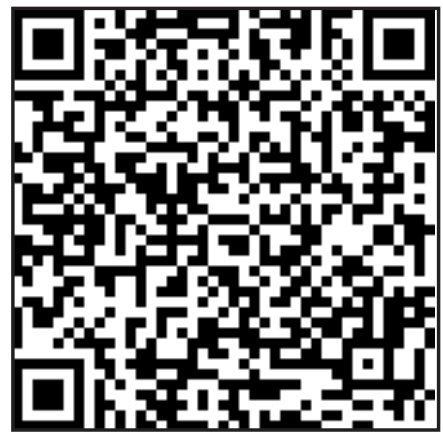

